\title{
Editorial
}

\section{Resource-Constrained Signal Processing in Sensor Networks}

\author{
Shuli Sun, ${ }^{1}$ Wendong Xiao, ${ }^{2}$ and Kung Yao ${ }^{3}$ \\ ${ }^{1}$ School of Electronics Engineering, Heilongjiang University, Harbin 150080, China \\ ${ }^{2}$ School of Automation and Electrical Engineering, University of Science and Technology Beijing, Beijing 100083, China \\ ${ }^{3}$ Electrical Engineering Department, University of California, Los Angeles, CA 90095-1594, USA \\ Correspondence should be addressed to Shuli Sun; sunsl@hlju.edu.cn
}

Received 13 February 2014; Accepted 13 February 2014; Published 17 March 2014

Copyright (C) 2014 Shuli Sun et al. This is an open access article distributed under the Creative Commons Attribution License, which permits unrestricted use, distribution, and reproduction in any medium, provided the original work is properly cited.

Sensor network is composed of spatially distributed sensor nodes to monitor the phenomenon of interest, where each sensor node takes measurements and transmits them to the data-processing center (fusion center). The main advantages of sensor network include its low cost, rapid deployment, self-organization, flexibility, and fault tolerance. Sensor networks, especially wireless sensor networks, have attracted significant research interests due to their wide applications and technological opportunities challenged by the limited resources, such as the battery-powered node energy and wireless bandwidth. Usually wireless channel in the network is also unreliable, which results in random transmission delays and packet losses when the sensor data are exchanged among nodes or sent to the data-processing center. Moreover, the quantization or compression of the sensor data is desired to tradeoff between the signal processing performance and the required network resources. It is very important to design collaborative signal processing algorithms and systems with network and sensor resource management under the uncertain network environment featured with the delayed, lost, or/and quantized data.

The main focus of this special issue will be on the new results of resource-constrained signal processing in sensor networks. It will provide an international platform for researchers to summarize the most recent development in the field. After a rigorous peer-reviewing process, 9 papers have been selected for publication. These papers cover the topics including compressed sensing, data compression, distributed estimation, consensus, and tracking.

In the paper entitled "IDMA-based compressed sensing for ocean monitoring information acquisition with sensor networks" by G. Liu and W. Kang, an interleave-division multiple-access- (IDMA-) based compressed sensing scheme is proposed for underwater sensor networks with applications to underwater environmental monitoring. The proposed scheme consists of three components: data sampling with randomly selected sensors, interleave-division multipleaccess of the sampled packets, and information recovery with the successfully accessed measurements after chipby-chip (CBC) multiuser detection (MUD). In the paper entitled "Data reduction with quantization constraints for decentralized estimation in wireless sensor networks" by Y. Weng, the unknown vector estimation problem is considered for bandwidth constrained wireless sensor network. Due to the power and communication bandwidth limitations, each sensor node must compress its data and transmit them to the fusion center. Both centralized and decentralized estimation frameworks are developed. The closed-form solution for the centralized estimation framework is proposed. The computational complexity of decentralized estimation problem is proven to be NP-hard and a Gauss-Seidel algorithm is also proposed to search for an optimal solution. In the paper entitled "Weighted measurement fusion quantized filtering with bandwidth constraints and missing measurements in sensor networks" by J. Ding et al., the estimation problem of a dynamic stochastic variable in a sensor network is studied, where the quantization of scalar measurement, the optimization of the bandwidth scheduling, and the characteristic of transmission channels are considered. Two weighted measurement fusion (WMF) quantized Kalman filters based on the quantized measurements arriving at the fusion center are presented for the imperfect channels with 
missing measurements in sensor networks. It is shown that they have the reduced computational cost and the same accuracy as the corresponding centralized fusion filter. The approximate solution for the optimal bandwidth-scheduling problem is given under a limited bandwidth constraint. In the paper entitled "Distributed fusion estimation for multisensor multirate systems with stochastic observation multiplicative noises" by F. Peng and S. Sun, a distributed fusion estimation algorithm is presented for a class of multisensor multirate systems with observation multiplicative noises. Sampling period of each sensor is uniform and the integer multiple of the state updates period. Moreover, different sensors have different sampling rates and observations of sensors are subject to the stochastic uncertainties of multiplicative noises. In the paper entitled "Average consensus analysis of distributed inference with uncertain Markovian transition probability" by W. I. Kim et al., the average consensus problem is studied for the distributed inference in a wireless sensor network under the Markovian communication topology with uncertain transition probability. A sufficient condition is presented for the average consensus of linear distributed inference algorithm. Based on linear matrix inequalities and numerical optimization, a design method is provided for fast distributed inference. In the paper entitled "IMM filter based human motion tracking using a wireless sensor network" by S. Zhang and W. Xiao, using low cost range wireless sensor nodes, an novel sensor selection algorithm is proposed for human tracking based on the interacting multiple model filter (IMM) techniques and considering both the tracking accuracy and the energy cost. In the paper entitled "Unknown clutter estimation by FMM approach in multitarget tracking algorithm" by $\mathrm{N}$. Lv et al., a multitarget tracking algorithm based on clutter model estimation is proposed to deal with severe bias caused by unknown and complex clutters. Multitarget likelihood function is established with FMM. In this frame, the algorithm of expectation maximum (EM) and Markov Chain Monte Carlo (MCMC) are both consulted in FMM parameters estimation. Furthermore, target number and multitarget states can be estimated precisely after the clutter model is fitted. In the paper entitled "Models and algorithms for tracking target with coordinated turn motion" by X. Yuan et al., firstly a number of widely used models are compared under the single model tracking framework, and the suggestions on the choice of models for different practical target tracking problems are given; then, in the multiple models (MM) framework, the algorithm based on expectation maximization (EM) algorithm is derived, including both the batch form and the recursive form. In the paper entitled "Self-similarity super resolution for resource-constrained image sensor node in wireless sensor networks" by Y. Wang et al., a self-similarity super resolution with low computation cost and high recovery performance is proposed. In the selfsimilarity image super resolution model, a small size sparse dictionary is learned from the image itself. The most similar patch is searched and specially combined during the sparse regulation iteration to preserve the detailed information.

The guest editors hope that this special issue can provide a snapshot of the latest advances in sensor networks and stimulate more research interest and efforts in sensor network research and development. They would like to acknowledge all authors for their efforts in submitting high-quality papers and are also very grateful to the reviewers for their professional contributions.

Shuli Sun

Wendong Xiao

Kung Yao 


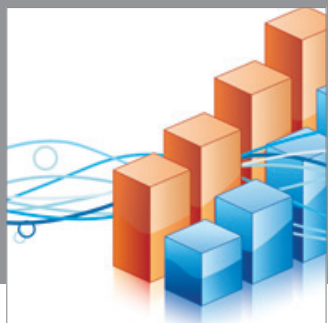

Advances in

Operations Research

mansans

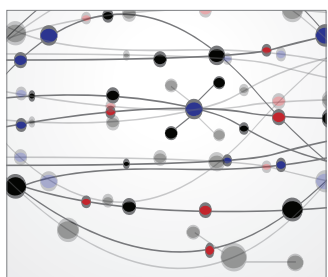

The Scientific World Journal
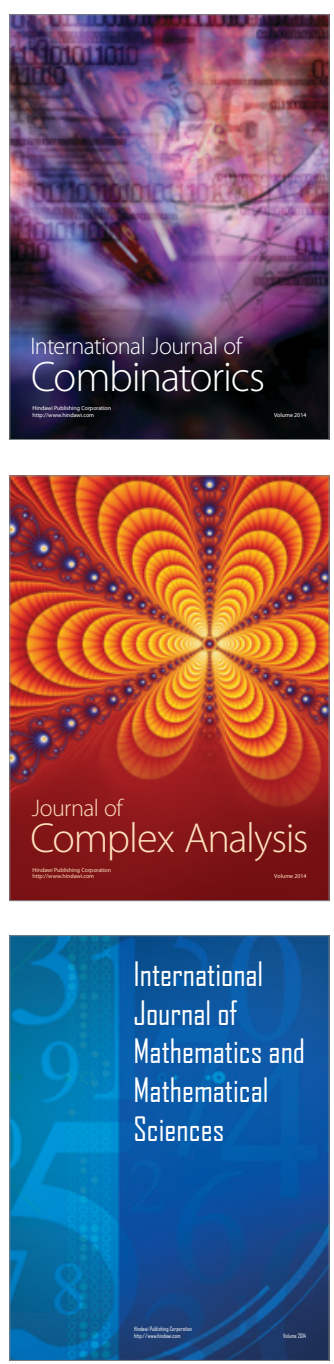
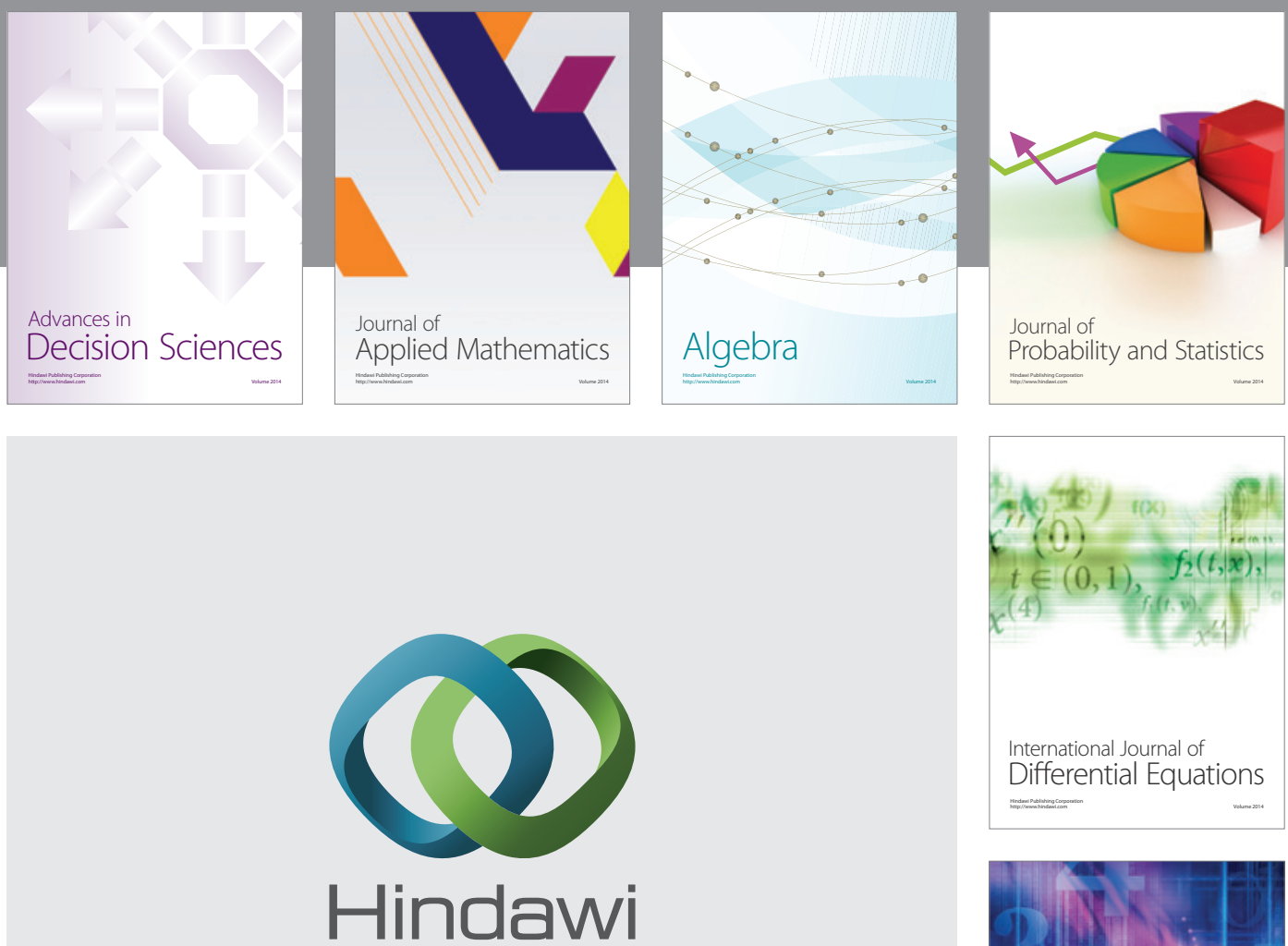

Submit your manuscripts at http://www.hindawi.com
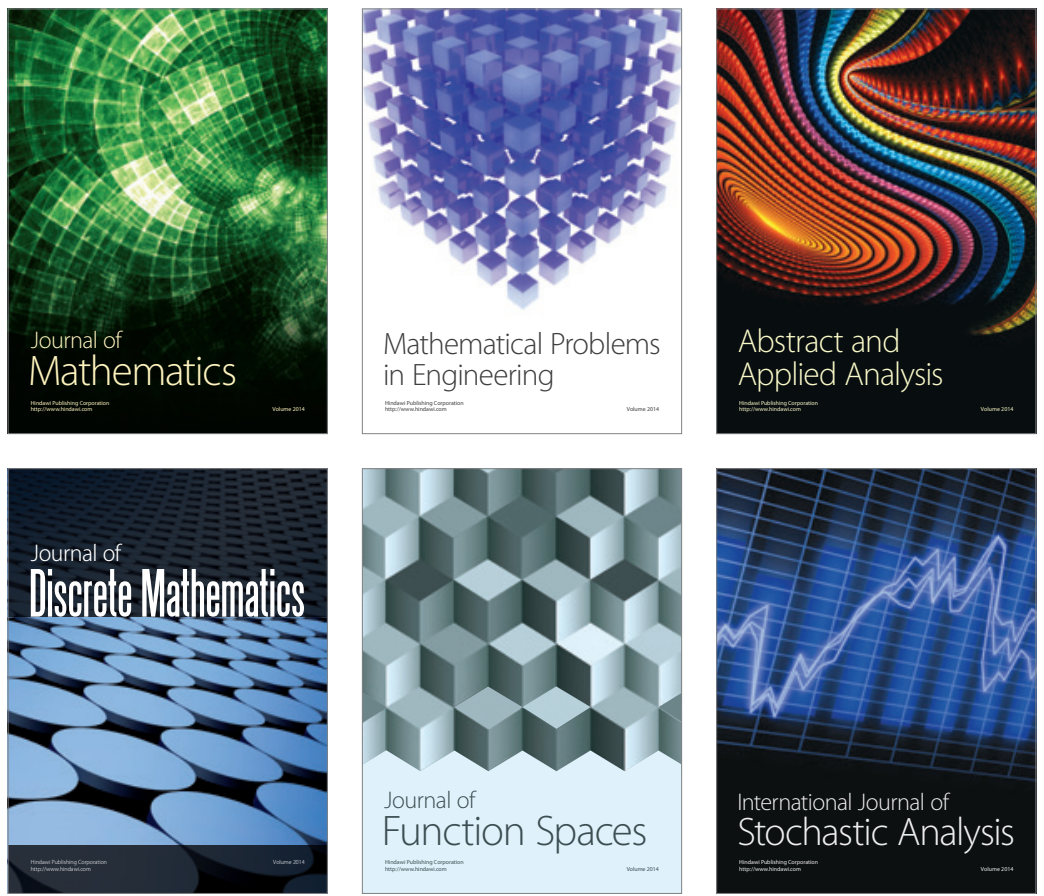

Journal of

Function Spaces

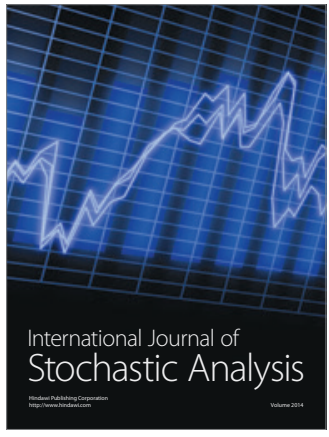

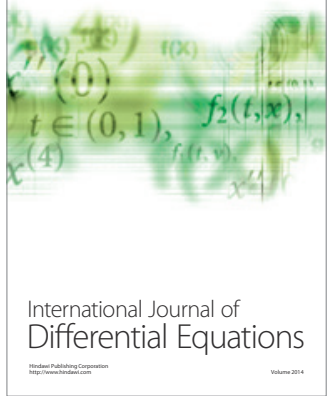
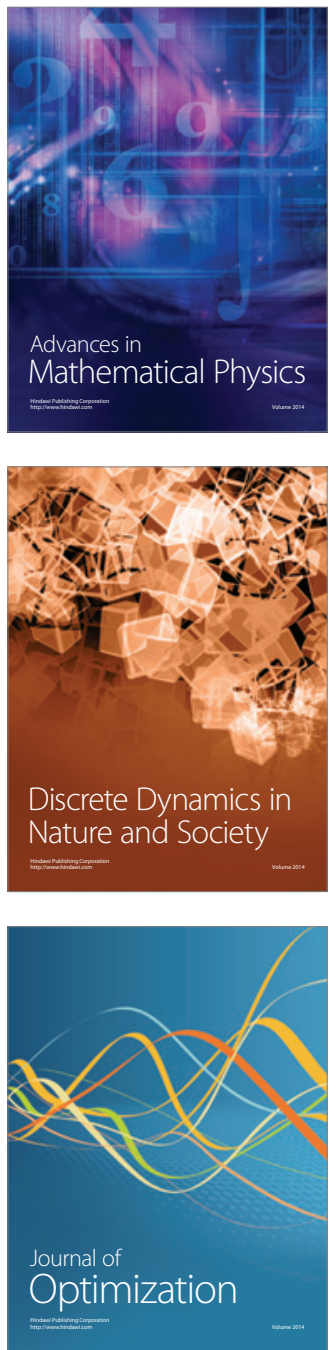